\title{
Estratégias para melhorar a comunicação entre o Dentista e o paciente adolescente: revisão integrativa da literatura
}

Strategies to improve communication between the Dentist and the adolescent patient: integrative literature review

Estrategias para mejorar la comunicación entre el Dentista y el paciente adolescente: revisión integradora de la literatura

\author{
Giovanna Burgos Souto Maior \\ ORCID: https://orcid.org/0000-0002-3361-5664 \\ Universidade de Pernambuco, Brasil \\ E-mail: giovannabsm@ @otmail.com \\ Trícia Murielly Andrade de Souza Mayer \\ ORCID: https://orcid.org/0000-0003-2640-4524 \\ Universidade de Pernambuco, Brasil \\ E-mail: triciamurielly@yahoo.com.br \\ Nayara Kelly de Melo Silva \\ ORCID: https://orcid.org/0000-0002-8842-9834 \\ Universidade Federal de Pernambuco, Brasil \\ E-mail: nayarak.melo@gmail.com \\ Viviane Colares \\ ORCID: https://orcid.org/0000-0003-2912-2100 \\ Universidade de Pernambuco, Brasil \\ E-mail: viviane.colares@upe.br
}

\begin{abstract}
Resumo
O objetivo deste estudo foi identificar e descrever possibilidades e estratégias para melhorar a comunicação entre o dentista e o paciente adolescente. Para tanto, realizou-se uma revisão integrativa da literatura mediante busca na base de dados MEDLINE/PubMed usando os descritores: adolescent, child, communication, health communication e dentist. A seleção dos estudos foi realizada por dois pesquisadores, de forma independente. Foram considerados estudos originais que incluíam participantes com idade entre 10 e 19 anos. Primeiro foi realizada a leitura de títulos e resumos, depois a seleção foi mediante leitura dos textos na íntegra. Por fim, seis estudos foram incluídos na revisão, correspondendo a literatura dos últimos oito anos. Um era um estudo transversal e os demais eram Ensaios Randomizados Controlados. Os estudos avaliaram estratégias em relação à comunicação verbal, escrita e virtual, como folhetos impressos, mapas mentais, formulários e recursos digitais. Como desfecho, obtiveram resultados positivos para diminuição da dor, e da ansiedade odontológica, e maior retenção de informações fornecidas pelo cirurgião-dentista. Diante disso, o cirurgião-dentista deve buscar meios adicionais para melhorar a comunicação com o paciente adolescente, e a literatura atual fornece algumas ferramentas com bons resultados, inclusive no que se refere ao atendimento do paciente com ansiedade odontológica. Incluir o resumo.
\end{abstract}

Palavras-chave: Adolescente; Comunicação; Comunicação em saúde; Dentista; Ansiedade ao tratamento odontológico.

\begin{abstract}
This study aimed to identify and describe strategies to improve communication between the dentist and adolescent patients. An integrative literature review was carried out in the MEDLINE/PubMed database using the following descriptors: adolescent, child, communication, health communication, and dentist. Retrieved studies were screened independently by two reviewers. This review included original studies with participants aged 10 to 19 years. Titles and abstracts were read first, followed by a full-text analysis for data extraction. A total of six studies were included, which covered the literature published in the last eight years. One study had a cross-sectional design, and the others were randomized controlled trials. Verbal, written and virtual communication strategies were described, such as printed brochures, mental maps, forms, and digital resources. Positive outcomes were observed concerning the reduction of pain and dental anxiety as well as greater retention of the information provided by the dentist. To conclude, dentists should make use of additional means to improve their communication with adolescent patients. Our review describes current evidence indicating that some tools are effective for this purpose and may be useful, for instance, in the care of patients with dental anxiety.
\end{abstract}


Keywords: Adolescent; Communication; Health communication; Dentist; Dental anxiety.

\section{Resumen}

El objetivo de este estudio fue identificar y describir estrategias para mejorar la comunicación entre el dentista y el paciente adolescente. Se realizó una revisión integradora de la literatura en la base de datos MEDLINE/PubMed utilizando los siguientes descriptores: adolescent, child, communication, health communication y dentist. La selección de los estudios fué realizada por dos revisores de forma independiente. Esta revisión incluyó estudios originales de participantes entre 10 a 19 años. Los títulos y resúmenes fueron examinados primero, seguido de un análisis de texto completo para la extracción de datos. Se incluyeron un total de seis estudios, correspondientes a la literatura publicada en los últimos ocho años, siendo un estudio transversal y los otros ensayos controlados aleatorios. Se describieron en los estudios estrategias de comunicación verbal, escrita y virtual, como folletos impresos, mapas mentales, formularios y recursos digitales. Resultados positivos fueron observados en cuanto a la reducción del dolor y la ansiedad dental, así como una mayor retención de la información transmitida por el dentista. En conclusión, los dentistas deben hacer uso de medios adicionales para mejorar su comunicación con los pacientes adolescentes. La evidencia actual indica que algunas herramientas son efectivas para este propósito y pueden ser útiles, por ejemplo, en el cuidado de pacientes con ansiedad dental.

Palabras clave: Adolescente; Comunicación; Comunicación en salud; Odontólogo; Ansiedad al tratamiento odontológico.

\section{Introdução}

A faixa etária da adolescência pode variar de acordo com circunstâncias culturais e históricas, mas para a Organização Mundial de Saúde (OMS) delimita-se cronologicamente dos 10 aos 19 anos de idade (WHO, 1986). Constantemente a adolescência é citada como um período de transição, no entanto essa visão tem favorecido o esquecimento das necessidades específicas desta população, sendo então necessário dar voz a esse público (Silva et al., 2021). Esta fase do desenvolvimento humano tem grande significado na vida de cada indivíduo, requerendo atenção e linguagem especial por parte dos profissionais da área da saúde. Além disso, do ponto de vista odontológico, é um período em que o risco de cárie dentária e de gengivite aumentam em decorrência de comportamentos negligentes com a saúde bucal (Bottan et al., 2015).

Nesse sentido, o cirurgião-dentista deve buscar estabelecer um vínculo com o paciente adolescente, levando em consideração o aspecto biológico, mas também avaliando características psicossociais e culturais. Esse vínculo se estabelece quando se conhece o universo dos adolescentes, acompanhando e demostrando interesse por suas preferências (Saadia \& Valencia, 2015). As interações dentista-paciente incluem dimensões emocionais, cognitivas, comportamentais e comunicativas, afetando o resultado geral dos cuidados em saúde bucal, especialmente para os pacientes com ansiedade odontológica. O paciente ansioso é geralmente mais vulnerável cognitivamente e mais sensível com relação às comunicações verbais e não verbais. Assim, quando ocorrem mal-entendidos durante o processo de comunicação, a ansiedade pode se elevar (Jaakola et al., 2014).

Uma forte relação dentista-paciente é importante porque influencia não só a ansiedade odontológica, como também os resultados de cuidados com a saúde, satisfação, confiança e adesão ao tratamento (Asan, Ye, Acharya, 2013; Kraft-Todd et al., 2017). Os cirurgiões-dentistas que têm boa interação com os pacientes foram vistos como mais competentes, e as habilidades de comunicação e informação foram classificadas como sendo os traços mais importantes desse profissional (Rodd et al., 2019; Bottan et al., 2009). Dessa forma, um modelo de comunicação centrado no paciente é recomendado, pois esse tipo de comunicação tem o foco nas necessidades, valores e desejos do paciente (Al Moebeeriekai, 2012; Okullo, Astrøm \& Haugejorden, 2004).

Diante disso, a comunicação eficiente e a empatia na relação dentista-paciente adolescente devem fazer parte das preocupações e rotina dos dentistas que atendem este público etário. No entanto, as abordagens usadas com crianças não são adequadas, e por outro lado os adolescentes não podem ser tratados como adultos. Assim, o objetivo deste estudo é identificar e descrever possibilidades e estratégias para melhorar a comunicação dentista-paciente adolescente. 


\section{Metodologia}

Este estudo trata-se de uma revisão integrativa, método que reúne, avalia e sintetiza os resultados de pesquisas sobre temática específica. As etapas seguidas na execução do estudo foram: elaboração da questão de pesquisa, busca na literatura dos estudos primários, extração de dados, avaliação dos estudos primários incluídos, interpretação dos resultados, apresentação da revisão (Mendes, Silveira \& Galvão, 2008).

\section{Estratégia de busca}

Elaborou-se a seguinte questão de pesquisa: "Quais são as estratégias encontradas na literatura para melhorar a comunicação entre o dentista e o paciente adolescente?". Para responder a essa pergunta, foi realizada uma busca ativa de informações na base de dados MEDLINE/PubMed. Para tanto, selecionou-se descritores controlados no MeSH Database e chegou-se à seguinte estratégica de busca: ((adolescent OR child) AND (communication OR "health communication")) AND (dentist). Realizou-se também uma busca manual na lista de referências dos artigos selecionados. As publicações foram inseridas no software Mendeley para gerenciar as referências e a última consulta às publicações foi realizada em novembro de 2019.

\section{Critérios de elegibilidade dos artigos}

Foram incluídos estudos originais que tenham avaliado a comunicação do cirurgião-dentista com o paciente adolescente, considerando os estudos que incluíam participantes com idades entre 10 e 19 anos, sem deficiências físicas ou intelectuais. A busca foi limitada a publicações nos idiomas inglês, português ou espanhol; e do tipo artigo científico. Não houve limite de tempo de publicação. Revisões, metanálises e relatos de caso foram excluídos.

\section{Seleção das publicações e extração dos dados}

O processo de análise para avaliação e seleção dos artigos foi realizado por dois pesquisadores, de forma independente, com posterior confronto dos resultados para obtenção dos textos selecionados por consenso. Em casos de divergências ou dúvidas quanto à inclusão dos artigos, houve a participação de um terceiro pesquisador avaliador. Os artigos repetidos foram considerados apenas uma vez. Então a seleção das publicações foi conduzida em duas fases: (1) leitura dos títulos e resumos e (2) análise qualitativa dos textos na íntegra. Esta metodologia seguiu as recomendações PRISMA (Principais Itens para Relatar Revisões Sistemáticas e Metanálises) (Moher et al., 2009).

\section{Resultados}

O fluxograma abaixo apresenta o processo de seleção dos trabalhos incluídos no estudo. Inicialmente, 560 artigos foram identificados no Pubmed por meio da busca por descritores. Desses, excluiu-se 490 após serem aplicados os filtros. Foram então avaliados 70 estudos por títulos e resumos, tendo sido excluídos 56 por não atenderem aos critérios de inclusão. Assim, 14 estudos tiveram os artigos lidos na íntegra, sendo seis selecionados para comporem a revisão de literatura (Figura 1). 
Figura 1: Fluxograma da estratégia de busca e seleção dos artigos via Pubmed.

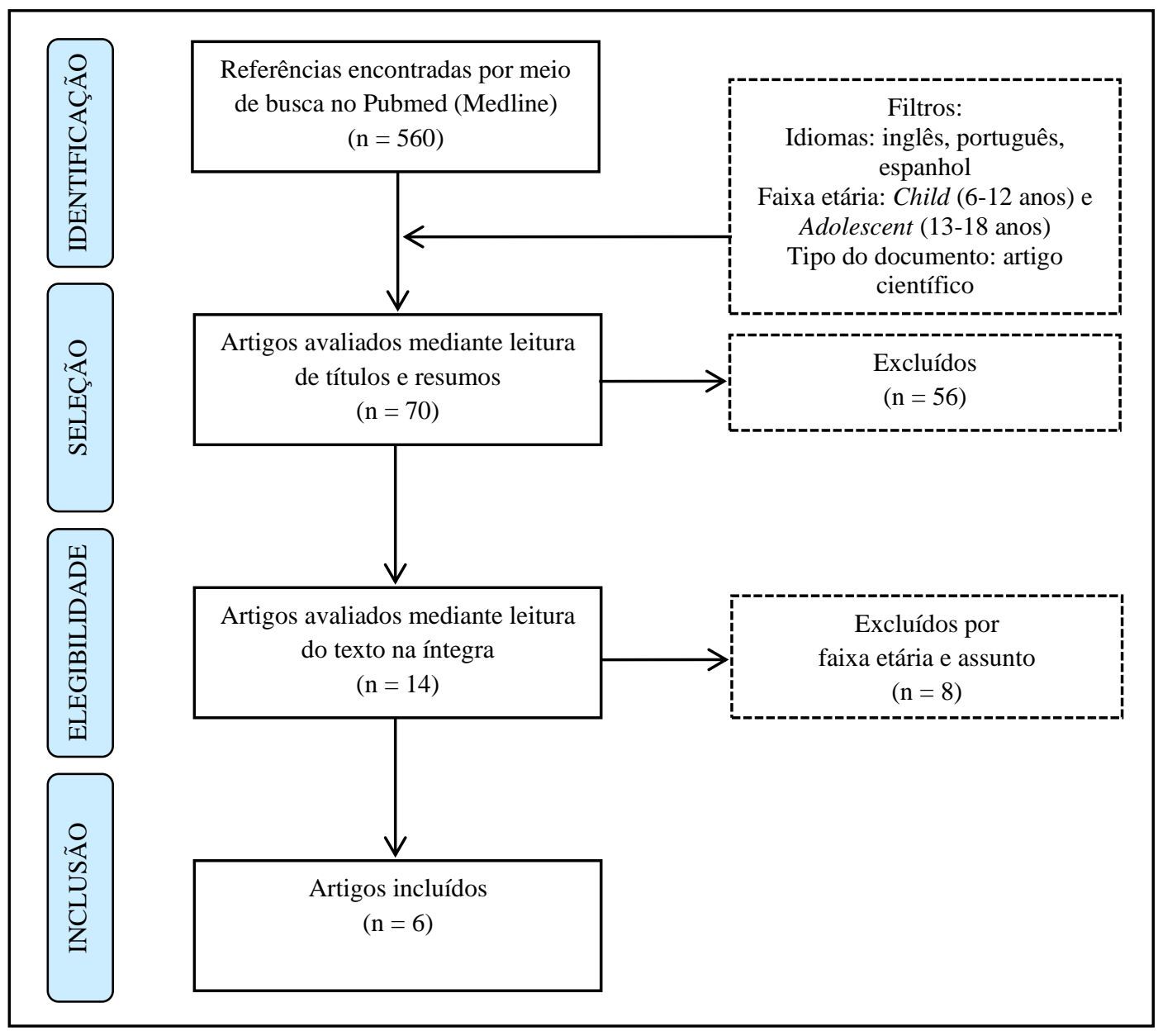

Fonte: Autores.

Quadro 1: Estratégias de comunicação entre dentistas e pacientes adolescentes.

\begin{tabular}{|c|c|c|c|c|c|}
\hline Autores, ano & País & $\underset{(n)}{\operatorname{Amostra}}$ & $\begin{array}{l}\text { Faixa } \\
\text { etária }\end{array}$ & $\begin{array}{l}\text { Estratégia de } \\
\text { comunicação }\end{array}$ & Principais conclusões \\
\hline $\begin{array}{l}\text { Carr et al., } \\
\quad 2012\end{array}$ & $\begin{array}{l}\text { Estados } \\
\text { Unidos }\end{array}$ & 80 & $12-18$ & $\begin{array}{l}\text { Orientações } \\
\text { por escrito, slides e } \\
\text { leitura pelo } \\
\text { ortodontista }\end{array}$ & $\begin{array}{l}\text { Não é necessária a leitura pelo } \\
\text { ortodontista. É suficiente uma escrita } \\
\text { legível, e apresentação de slides com as } \\
\text { principais informações constando no } \\
\text { início e ao final da apresentação. }\end{array}$ \\
\hline $\begin{array}{l}\text { Keith et al., } \\
2013 \\
\end{array}$ & $\begin{array}{l}\text { Estados } \\
\text { Unidos } \\
\end{array}$ & 39 & $10-18$ & $\begin{array}{c}\text { Mensagens virtuais } \\
\text { por SMS } \\
\end{array}$ & Redução da dor \\
\hline Jones, 2015 & $\begin{array}{c}\text { Nova } \\
\text { Zelândia }\end{array}$ & 128 & $10-13$ & $\begin{array}{c}\text { Programa de } \\
\text { computador (eSAID) }\end{array}$ & Redução de ansiedade \\
\hline $\begin{array}{l}\text { Yee; Jones; } \\
\text { Hosey, } 2017\end{array}$ & $\begin{array}{l}\text { Reino } \\
\text { Unido }\end{array}$ & 56 & $8-13$ & $\begin{array}{l}\text { Programa de } \\
\text { computador } \\
\text { (e-SAID) }\end{array}$ & Redução de ansiedade \\
\hline $\begin{array}{l}\text { Ahn et al., } \\
2019\end{array}$ & $\begin{array}{l}\text { Reino } \\
\text { Unido }\end{array}$ & 88 & $10-19$ & $\begin{array}{l}\text { Orientações por } \\
\text { escrito e mapas } \\
\text { mentais }\end{array}$ & $\begin{array}{l}\text { Mapa mental apresentou melhor } \\
\text { retenção de informação }\end{array}$ \\
\hline $\begin{array}{l}\text { Rodd et al., } \\
\quad 2019\end{array}$ & $\begin{array}{l}\text { Reino } \\
\text { Unido }\end{array}$ & 105 & $9-16$ & $\begin{array}{l}\text { Formulário impresso } \\
\text { (Message to Dentist - } \\
\text { MTD) }\end{array}$ & $\begin{array}{l}\text { Redução dos escores de preocupação e } \\
\text { dor }\end{array}$ \\
\hline
\end{tabular}

Fonte: Autores.

O Quadro 1 relaciona os dados extraídos dos seis artigos selecionados. Esses artigos foram publicados nos últimos 
oito anos, um era um estudo transversal (Rodd et al., 2019) e os demais eram Ensaios Randomizados Controlados (ERC). Esses estudos avaliaram estratégias em relação à comunicação verbal, escrita e virtual. Como desfecho, obtiveram resultados como melhor retenção de informações prestadas, diminuição da dor e da ansiedade odontológica. Três estudos avaliaram a comunicação em situação de tratamento ortodôntico e três no contexto do atendimento odontopediátrico.

\section{Discussão}

Alguns estudos avaliaram a comunicação dos cirurgiões-dentistas com seus pacientes adolescentes, principalmente em relação à retenção de informações passadas durante as consultas odontológicas (Carr et al., 2012; Ketih et al., 2013; Ahn et al., 2019). Carr et al. (2012) avaliaram a capacidade de os adolescentes compreenderem e lembrarem das informações fornecidas pelos ortodontistas em relação aos cuidados, riscos e limitações do tratamento ortodôntico. Para tanto, dividiram os adolescentes em dois grupos, onde ambos receberam as informações por meio de um documento impresso. Esse continha menos terminologia odontológica, estava escrito na voz ativa, com fonte em tamanho maior e espaçamento equilibrado. Além disso, todos os participantes assistiram a uma apresentação de slides, mas em apenas um dos grupos o ortodontista revisou verbalmente os itens do documento. No entanto, não houve diferença entre os grupos e os autores concluíram que usando esse método (escrita legível e apresentação de slides), não é necessária a leitura pelo ortodontista. Porém, apesar de os adolescentes terem recordado as informações, isso não significava que eles as compreenderam. Assim, os autores orientam que as principais informações devem ser tratadas no início e ao final da apresentação, pois nesses momentos são mais bem compreendidas.

No estudo desenvolvido por Ahn et al. (2019), também foi avaliada a retenção de informação pelo paciente adolescente, mas tanto a curto quanto a longo prazo, através da comparação de três formas de fornecer informações sobre o tratamento ortodôntico. O estudo comparou orientações escritas em um folheto com dois tipos de mapas mentais (diagrama que ajuda na organização de informações). Foi visto que o mapa mental é uma alternativa significativamente melhor que os folhetos para a retenção de informações, não havendo diferença estatística entre um mapa mental preparado previamente e outro personalizado, construído pelo paciente. Porém, este estudo não fez comparação com a comunicação verbal, como foi realizado no estudo de Carr et al. (2012).

Nesse sentido, no contexto da comunicação com o adolescente, para Keith et al. (2013) a palavra escrita seria mais importante que a palavra falada, concordando com os resultados de Carr et al. (2012). Neste estudo foram enviadas mensagens telefônicas para pacientes do grupo de intervenção que iniciaram tratamento ortodôntico, demostrando preocupação e fornecendo instruções, enquanto o grupo controle não recebeu nenhuma mensagem. Como resultado, foi visto que os pacientes do grupo de intervenção relataram um menor nível de dor após instalação do aparelho ortodôntico. Este estudo, além de frisar a importância da comunicação escrita, destaca os meios virtuais para comunicação com adolescentes e até adultos (Keith et al., 2013).

Diante disso, dois estudos avaliaram um programa de computador chamado e-SAID (Survey of Anxiety and Information for Dentists), que tem como propósito facilitar a comunicação entre o dentista e o paciente, reduzindo a ansiedade deste (Jones, 2015; Yee, Jones \& Hosey, 2017). Trata-se de um questionário, o primeiro do seu tipo, contendo 25 perguntas, onde a maioria é respondida por meio de uma escala de faces de 7 pontos. Há também questões abertas com espaço ilimitado para que o adolescente digite sua resposta, pergunta ou comentário. O e-SAID é respondido no computador na sala de espera do consultório odontológico, e após isso é impresso um relatório que a criança/adolescente entrega ao dentista. Assim, essa ferramenta fornece aos dentistas uma maneira sistemática e qualitativa de “ouvir" seus pacientes, agrupando as solicitações, sentimentos, preferências de enfrentamento e ansiedade dos pacientes em um único relatório impresso (Jones, 2015).

Além disso, o e-SAID visa que crianças e adolescentes percebam por meio das questões, mesmo antes de adentrar a sala de atendimento, que o dentista se importa com seus sentimentos em relação à consulta. O próprio nome do instrumento, 
"SAID" sugere que o paciente se expresse (Jones \& Huggins, 2014; Jones, 2015). Nesse sentido, a empatia percebida pode ser uma importante estratégia, onde a principal característica da relação terapêutica dentista-paciente não é se há uma demonstração de empatia, mas se essa empatia é percebida no relacionamento. Ou seja, o paciente precisa acreditar que está recebendo uma resposta empática do dentista (Jones \& Huggins, 2014).

Os resultados desses dois estudos que avaliaram o e-SAID demonstraram que seu uso foi capaz de reduzir a ansiedade odontológica. No primeiro estudo houve uma redução de 5,5\% na ansiedade entre as medidas realizadas antes e após a aplicação do e-SAID, comparado a um aumento de 2,4\% na ansiedade para os participantes do grupo controle (Jones, 2015). Já no segundo estudo, o nível de ansiedade do grupo de intervenção reduziu em 33\%, enquanto no grupo controle aumentou em 16\%, havendo diferença estatística entre os grupos. Diferentemente do ensaio clínico anterior, este avaliou se os indivíduos se sentiram beneficiados com o uso do e-SAID, a satisfação com a consulta odontológica e a cooperação dos pacientes com a consulta odontológica. No entanto, não houve diferença entre os grupos em nenhum desses aspectos (Yee, Jones \& Hosey, 2017).

Outra nova ferramenta com o objetivo ajudar na comunicação entre o paciente jovem com ansiedade odontológica e o dentista é o 'Message to Dentist' (MTD). Trata-se de um formulário impresso entregue ao paciente antes do início do tratamento com o objetivo de incentivá-lo a informar o dentista sobre suas preocupações específicas e estratégias planejadas de enfrentamento. O MTD também é utilizado ao final do tratamento para estimular a autorreflexão e construir memórias positivas. Como resultados observou-se que, ao concluir o tratamento, os pacientes relataram estar significativamente menos preocupados, e reduziram significativamente o escore de dor. A estratégia de enfrentamento mais popular foi a distração, por meio de música ou audiolivro, e os pacientes esperavam que a equipe odontológica os ouvisse e contasse o que estava acontecendo. Além disso, eles queriam se sentir no controle, podendo pedindo ao dentista para interromper o tratamento quando necessário. Dessa forma, o MTD se mostrou um meio eficaz de facilitar a comunicação entre jovens ansiosos e a equipe odontológica, permitindo-lhes identificar as preocupações deles e elaborar planos de enfrentamento personalizados (Rodd et al., 2019).

\section{Conclusão}

O cirurgião-dentista deve buscar meios adicionais para melhorar a comunicação com o paciente adolescente, e a literatura atual fornece algumas ferramentas com bons resultados, inclusive no que se refere ao atendimento do paciente com ansiedade odontológica. Nesse sentido, além da comunicação verbal, folhetos impressos escritos de forma clara e objetiva, mapas mentais previamente elaborados, formulários e recursos digitais, se apresentam como recursos que podem ser utilizados para a melhor interação profissional-paciente. No entanto, ainda há poucos estudos nesse campo, por isso mais pesquisas que se dediquem a criar e avaliar ferramentas ou estratégias para melhorar a comunicação entre dentista e adolescente são necessárias.

\section{Referências}

Ahn, J. H., Power, S., Thickett, E., Andiappan, M., \& Newton, T. (2019). Information retention of orthodontic patients and parents: A randomized controlled trial. American journal of orthodontics and dentofacial orthopedics, 156(2), 169-177.

Al-Mobeeriek A. (2012). Dentist-patient communication as perceived by patients in Riyadh, Saudi Arabia. International journal of occupational medicine and environmental health, 25(1), 89-96.

Asan, O., Ye, Z., \& Acharya, A. (2013). Dental care providers' and patients' perceptions of the effect of health information technology in the dental care setting. Journal of the American Dental Association (1939), 144(9), 1022-1029.

Bottan, E. R., Oglio, J. D., Silveira, E. G., \& Araújo, S. M. (2009). Cirurgião-dentista ideal: perfil definido por crianças e adolescentes. RSBO Revista SulBrasileira de Odontologia, 6(4), 381-386. 
Research, Society and Development, v. 10, n. 3, e33910312973, 2021

(CC BY 4.0) | ISSN 2525-3409 | DOI: http://dx.doi.org/10.33448/rsd-v10i3.12973

Bottan, E. R., Viroetti, A. J., Santi, D. G., \& Silveira, E. G. (2015). Percepção de adolescentes sobre as competências essenciais ao cirurgião-dentista. Arquivos em Odontologia, 51(3), 145-151.

Carr, K. M., Fields, H. W., Jr, Beck, F. M., Kang, E. Y., Kiyak, H. A., Pawlak, C. E., \& Firestone, A. R. (2012). Impact of verbal explanation and modified consent materials on orthodontic informed consent. American journal of orthodontics and dentofacial orthopedics, 141(2), 174-186.

Jaakkola, S., Lahti, S., Räihä, H., Saarinen, M., Tolvanen, M., Aromaa, M., Sillanpää, M., Suominen, S., Mattila, M. L., \& Rautava, P. (2014). Dental fear affects adolescent perception of interaction with dental staff. European journal of oral sciences, 122(5), 339-345.

Jones, L. M., \& Huggins, T. J. (2014). Empathy in the dentist-patient relationship: review and application. The New Zealand dental journal, $110(3), 98-104$.

Jones L. (2015). Validation and randomized control trial of the e-SAID, a computerized paediatric dental patient request form, to intervene in dental anxiety. Child: care, health and development, 41(4), 620-625.

Kraft-Todd, G. T., Reinero, D. A., Kelley, J. M., Heberlein, A. S., Baer, L., \& Riess, H. (2017). Empathic nonverbal behavior increases ratings of both warmth and competence in a medical context. PloS one, 12(5).

Keith, D. J., Rinchuse, D. J., Kennedy, M., \& Zullo, T. (2013). Effect of text message follow-up on patient's self-reported level of pain and anxiety. The Angle orthodontist, 83(4), 605-610.

Mendes, K. D. S., Silveira, R. C. C. P., \& Galvão, C. M. (2008). Revisão integrativa: método de pesquisa para a incorporação de evidências na saúde e na enfermagem. Texto \& Contexto - Enfermagem, 17(4), 758-764.

Moher D, Liberati A, Tetzlaff J, \& Altman, D. G, The PRISMA Group (2009) Preferred Reporting Items for Systematic Reviews and Meta-Analyses: The PRISMA Statement. PLoS Med, 6(7).

Okullo, I., Astrøm, A. N., \& Haugejorden, O. (2004). Influence of perceived provider performance on satisfaction with oral he alth care among adolescents Community dentistry and oral epidemiology, 32(6), 447-455.

Rodd, H., Timms, L., Noble, F., Bux, S., Porritt, J., \& Marshman, Z. (2019). 'Message to Dentist': Facilitating Communication with Dentally Anxious Children. Dentistry journal, 7(3), 69.

Saadia, M., \& Valencia, R. (2015). Communicating with the New Generations. The Challenge for Pediatric Dentists. The Journal of clinical pediatric dentistry, 39(4), 297-302.

Silva, M. W., Franco, E. C. D, Gadelha, A. K. O. A., Costa, C. C., \& Sousa, C. F. (2021) Adolescência e Saúde: significados atribuídos por adolescentes Research, Society and Development, 10(2), p. e27510212482.

WHO Study Group on Young People and 'Health for All by the Year 2000' \& World Health Organization. (1986). Young people's health - a challenge for society: report of a WHO Study Group on Young People and "Health for All by the Year 2000" [meeting held in Geneva from 4 to 8 June 1984]. World Health Organization.

Yee, R., Jones, L. M., \& Hosey, M. T. (2017). What the child "SAID" to the dentist: A UK randomized controlled trial. Child: care, health and development, 43(6), 926-932. 